\title{
Experimental alteration of the phenotype of animal cells in vivo
}

\author{
CHARLES B. HUGGINS \\ From the Ben May Laboratory for Cancer Research, The University of Chicago, Chicago, Illinois
}

Why does one cell differ from another, a brain cell from a liver cell? Why do the cells of an animal become programmed and thereafter retain their individual identity? Under normal circumstances all somatic cells of a multicellular organism possess an identical genome and, consequently, an identical potential to synthesize every kind of molecule produced by the organism at every stage of its development. Yet normally less than $1 \%$ of the genetic potential of the cell is expressed. The cells of the organism have become programmed selectively and they remain throughout life highly defined in identity. The normal cell of adult animals zealously protects the integrity of its phenotype and its alteration generally leads to cell death or, less often, to cancer. The sorting out of form and function is denoted 'differentiation', which is generally pictured as turning on or off of DNA in a chromosomal array by specific regulatory molecules.

This paper is concerned with the physiology and biochemistry of differentiation and the phenotype of the cancer cell. I shall describe methods of utter simplicity to cause: (1) the transformation of normal cells into normal cells of another type in adult animals and (2) to change, rapidly and invariably, the program of normal cells into neoplasms.

The change from normal into cancer cell can be brought about in many kinds of cells by many different experimental procedures. In animals the change of a normal cell into a normal cell of a different sort has been accomplished experimentally only in the case of a single cell species-the fibroblast (mesenchyma; stem cell of connective tissue).

To embryologists the endpoint of an inductive interaction is phenotypic expression of responding cells. In postfoetal life, long after embryonic differentiation has ceased, some cells of the connective tissue still remain undifferentiated. These primitive dormant cells possess a remarkable attribute-they can be induced by experimental means readily to assume a new program which thereafter is perpetuated permanently.

There are two distinctive methods to alter the phenotype of the competent fibroblasts. These consist of bringing them in association with, respectively, transforming epithelium or demineralized matrix of bone and tooth. Approximation of transformant and responding fibroblast initiates a series of interconnected biochemical reactions that yield highly distinctive products. The net result is permanent alteration of the phenotype of the fibroblast.

\section{Transforming Epithelium}

The remarkable mutability of fibroblasts which permits radical changes of phenotypic expression was found out in the dog (Huggins, 1930). The surgical approximation of certain epithelia with fascia of the trunk or limb evoked large amounts of bone which invariably were evident in 12 days; it is noteworthy that cartilage was not observed. In autologous transplants the ossicle which had been created by the transforming epithelium persisted for more than two years. Experimental approximation of the reactive cells had set in motion a train of great events which would not have occurred in absence of contact of the reacting tissues.

Three sorts of epithelium are osteogenic: urinary tract (Huggins, 1930), gallbladder (Huggins and Sammet, 1933), and seminal vesicle (Huggins, 1969). Epithelial osteogenesis can be evoked with regularity in dog and guinea pig (Huggins, 1969) but rather infrequently in the rat. Bone does not occur in the normal urinary bladder despite the fact that its submucosal fibroblasts lie in close proximity to the potentially powerful transforming epithelium. These fibroblasts are incompetent to respond by changing their phenotype.

\section{Transformation of Fibroblasts by Collagens}

Urist (1965) discovered that decalcified lyophilized matrix of bone or tooth can act as an inductive substrate' since it evokes differentiation of fibroblasts into cartilage and, later, bone and bone marrow. 
Urist, Dowell, Hay, and Strates (1968) implanted segments of the acid-insoluble residue of bone or tooth into surgically prepared pockets in the muscle of rabbits and found that bone formed by endochondral ossification in 24 to 26 days. Chondrogenesis occurs in cell culture (Urist and Nogami, 1970) as well as in vivo.

The experimental method for transformation of fibroblasts by matrices of bone or tooth was simplified by the use of acid-insoluble demineralized powders of bone or tooth. The preparation of the dry transforming collagens was complete in six hours and they retained full potency at room temperature for as long as two years. The method of bioassay was simplified by implanting the dry powders of transforming collagens subcutaneously in rats. Convenient quantitative assays of transformation are enzymic (Huggins and Urist, 1970) and radiochemical (Reddi and Huggins, 1972).

There are many powders in this world. The transforming collagens are the only powders which can change the phenotype of normal cells without the intervention of cancer. Remarkably the transformants are insoluble in water, strong acids, salt solutions, or lipids. The transforming collagens are rapidly inactivated by dilute alkali or formaldehyde.

The transformation of fibroblasts in adult animals is reminiscent of the process of differentiation in embryonic life. Transformability is a regulated function that is inactive in bone in its native state; it becomes active when bone minerals are removed.

Powders of acid-insoluble bone matrix had an intense chemotactic attraction for fibroblasts, which aggregated around the transformant to encapsulate it within 24 hours to form the transformation plaque; it is noteworthy that giant cells and inflammations were absent. The plaque had a thick centre and a thin periphery. Transformation occurred exclusively in the centre of the plaque, never in its rim.

The technique provided a convenient method to produce de novo large masses of cartilage, bone, and erythropoietic bone marrow in the adult rat. Rather coarse powders (particle size 74-420 $\mu \mathrm{m}$ ) were highly efficient in creating large quantities of the transformation products.

There was a strict time table of transformation that was reproduced repeatedly in our experiments. It was noteworthy that transformation plaques formed in 486 consecutive transplantations in our experiments.

In the present experiments, chondrogenesis was an unstable transformation. Chondroblasts appeared on day 5 and large masses of cartilage were present on days 7 and 8 . Chondrolysis began on day 9 and in most cases cartilage had vanished before day 18 .
Chondrolysis and the subsequent replacement of cartilage by bone began with the invasion of the transformation plaque by capillaries.

The change of phenotype of fibroblast to osteoblast was a stable transformation. Living bone, with haemopoietic bone marrow, was found on day 700 , long after the transformant had disappeared.

The optimal time for harvest of newly created cartilage, devoid of bone, was day 7-8. To obtain bone without cartilage, days 18-21 were advantageous for harvest. Because of the large content of erythropoietic bone marrow, the newly formed ossicles were vivid red from days 18 to $700+$.

It was noteworthy that the concentration of ${ }^{35} \mathrm{~S}$ was appreciable in the sulphur-containing macromolecules of the transformation plaques before cartilage cells were observed.

The parietal bone of the calvarium develops in the embryo by membranous ossification without an intervening stage of cartilage. But, transplants of acid-insoluble residue prepared from parietal bones yielded a typical endochondral ossification.

\section{Rapid Induction and Cure of Experimental Leukaemias}

Under simple conditions a single feeding (Huggins, Grand, and Brillantes, 1961) or intravenous injection (pulse-dose) of 7,12-dimethylbenz(a)anthracene (7,12-DMBA) evoked mammary cancer in every rat whereas leukaemia arose rarely. By rearrangement of the experimental conditions leukaemia was induced in high yield but with low incidence of mammary cancer.

The leukaemogenic action of polycyclic aromatic hydrocarbons was discovered by Morton and Mider (1938) by repeatedly painting the skin of mice with 3-methylcholanthrene. The incidence of leukaemia approached $100 \%$ with a mean latent period of detection of 86 days; in the predominant type of leukaemia, thymus, spleen, and lymph nodes were enlarged.

In the experiments of Huggins and Sugiyama (1966), groups of rats were injected intravenously with four pulse doses of a lipid emulsion of 7,12DMBA at biweekly intervals beginning at age 27 days; every rat developed leukaemia. Most of the leukaemias were erythroblastic in character associated with massive leukaemic involvement of liver, spleen (Bird and Huggins, 1971), and bone marrow (Bird, 1972); the thymus was not enlarged. The haemograms were not abnormal until a late stage of leukaemia and then leukocytosis was commonly slight or moderate. On histological examination of the liver, large immature tumour cells were found increasingly to invade hepatic sinusoids until the 
sinusoidal endothelium was completely replaced by them.

Regression followed hypophysectomy in $31 \%$ of rats with erythroblastic leukaemia (Huggins and Oka, 1972) induced by a set of pulse-doses of 7,8 , 12-trimethylbenz(a)anthracene (7,8,12-TMBA).

A large proportion of rats with experimentally induced erythroblastic leukaemia had a consistent abnormality consisting of trisomy of the longest telocentric chromosome (Sugiyama, Kurita, and Nishizuka, 1967). The leukaemia cells with the nonrandom trisomy were erythroblasts.

Rees, Majumdar, and Shuck (1970) found that 24 hours after a single pulse-dose of 7,12-DMBA or $7,8,12-\mathrm{TMBA}$ about half of the metaphase cells in bone marrow had chromosomes with breaks or gaps. These aberrations were not random; certain pairs of chromosomes were involved to an extent greater than expected on the basis of their size and number. Rees and his colleagues found distinctive karyotypic abnormalities involving the $\mathrm{C}-1$ chromosome in half of the leukaemic rats whereas abnormalities of this kind were not observed in the bone marrow of nonleukaemic control rats which had been injected with 7,12-DMBA.

It will be recalled that Nowell and Hungerford (1960) discovered the presence of a consistent chromosome abnormality $\left(\mathrm{Ph}^{1}\right.$ chromosome) in human granulocytic leukaemia.

\section{Phenotype of the Cancer Cell}

As a working hypothesis we regard cancer as a specific reaction to injury. The cancer cell has a distinguishable phenotype. In general, the function of cancer cells is characterized by a decrease in differentiated function and an increased metabolic autonomy.

Greenstein (1947) made a comprehensive study of the profiles of enzymes in malignant neoplasms from which an important generalization was formulated. Greenstein's law states: 'No matter how or from which tissue malignant tumours arise, they more nearly resemble each other chemically than they do normal tissues or than normal tissues resemble each other.'

Warburg (1930) discovered that cancer cells grow as facultative anerobes. There is a high rate of formation of lactic acid both in the presence and absence of oxygen. Cancer behaves in respiration not like muscle but like yeast.

\section{Summary}

Three simple methods are presented whereby the phenotype of cells in living animals is altered.

1 Responding fibroblasts change to osteoblasts by bringing them in contact with transforming epithelium.

2 Responding fibroblasts change to chondroblasts after contact with matrix of bone or tooth.

3 Stem cells of the reticuloendothelial system change to erythroblastic leukaemia after exposure to highly active polycyclic aromatic hydrocarbons.

This work was supported by grants from the American Cancer Society, The Jane Coffin Childs Memorial Fund for Medical Research, and the US Public Health Service, National Institutes of Health (no CA11603).

\section{References}

Bird, C. (1972). Leukemia induced by 7,8,12-Trimethylbenz(a)anthracene in rats. II. Changes in bone marrow. J. nat. Cancer Inst., 48, 429-439.

Bird, C., and Huggins, C. (1971). Leukemia evoked with 7,8,12trimethylbenz(a)anthracene in rats. $I$. Changes in spleen and thymus. J. exp. Med., 134, 1285-1297.

Greenstein, J. P. (1947). Biochemistry of Cancer. Academic Press, New York.

Huggins, C. B. (1930). Influence of urinary tract mucosa on the experimental formation of bone. Proc. Soc. exp. Biol. (N.Y.), 27, 349-351.

Huggins, C. B. (1969). Epithelial osteogenesis: a biological chain reaction. Proc. Amer. philosoph. Soc., 113, 458-463.

Huggins, C. B., Grand, L. C., and Brillantes, F. P. (1961). Mammary cancer induced by a single feeding of polynuclear hydrocarbons and its suppression. Nature (Lond.), 189, 204-207.

Huggins, C. B., and Oka, H. (1972). Regression of stem-cell erythroblastic leukemia after hypophysectomy. Cancer Res., 32, 239-242.

Huggins, C. B., and Sammet, J. F. (1933). Function of the gall bladder epithelium as an osteogenic stimulus and the physiological differentiation of connective tissue. J. Exp. Med., 58, 393-400.

Huggins, C. B., and Sugiyama, T. (1966). Induction of leukemia in rat by pulse doses of 7,12-Dimethylbenz(a)anthracene. Proc. nat. Acad. Sci. (Wash.), 55, 74-81.

Huggins, C. B., and Urist, M. R. (1970). Dentin matrix transformation: rapid induction of alkaline phosphatase and cartilage. Science, 167, 896-897.

Morton, J. J., and Mider, G. B. (1938). The production of lymphomatosis in mice of known genetic constitution. Science, 87, 327-328.

Nowell, P. C., and Hungerford, D. A. (1960). Chromosome studies on normal and leukemic human leukocytes. J. nat. Cancer Inst., 25, 85-109.

Reddi, A. H., and Huggins, C. B. (1972). Biochemical sequences in the transformation of normal fibroblasts in adolescent rats. Proc. nat. Acad. Sci. (Wash.), 69, 1601-1605.

Rees, E. D., Majumdar, S. K., and Shuck, A. (1970). Changes in chromosomes of bone marrow after intravenous injections of 7,12-Dimethylbenz(a)anthracene and related compounds. Proc. nat. Acad. Sci. (Wash.), 66, 1228-1235.

Sugiyama, T., Kurita, Y., and Nishizuka, Y. (1967). Chromosome abnormality in rat leukemia induced by 7,12-dimethylbenz(a)anthracene. Science, 158, 1058-1059.

Urist, M. R. (1965). Bone formation by autoinduction. Science, 150, 893-899.

Urist, M. R., Dowell, T. A., Hay, P. H., and Strates, B. S. (1968). Inductive substrates for bone formation. Clin. Orthop., 59, 59-96.

Urist, M. R., and Nogami, H. (1970). Morphogenetic substratum for differentiation of cartilage in tissue culture. Nature (Lond.), 225, 1051-1052.

Warburg, O. (1930). The Metabolism of Tumours. (Translated by F. Dickens). Constable, London. 